\title{
Planning of Medium-Voltage Electric Power Distribution Systems through a Scatter Search Algorithm
}

\author{
S. G. B d. Pádua, A. M. Cossi and J. R. S. Mantovani, Member, IEEE
}

\begin{abstract}
In this paper the problem of Planning Electric Power Distribution Systems (PEPDS) of Medium Voltage is formulated as a mixed integer nonlinear programming (MINLP) model. For solving this model the scatter search (SS) algorithm is proposed, an evolutionary search technique which according to the specialized literature has not yet been used for solving this problem. The main contribution of this work is the description of the five basic stages of SS for the PEPDS problem. The method was applied to 54 and 417 busbars from testing systems found in the specialized literature.
\end{abstract}

Keywords - Distribution System, Planning, Scatter Search, Combinatorial Optimization.

\section{INTRODUÇÃO}

A O longo dos últimos anos as meta-heurísticas têm se destacado como técnicas atraentes para solução do problema de planejamento de sistemas de distribuição de energia elétrica (PSDEE) de grande e médio porte, encontrando soluções otimizadas de boa qualidade. A metaheurística Tabu Search (TS) foi utilizada na solução do problema de planejamento otimizado de sistemas de distribuição em [1], [2] e [3], Algoritmos Genéticos (AG) foram empregados em [4], [5], [6] e [7], Ant Colony System em [8] e uma adaptação do algoritmo Sistema Imune Artificial em [9].

Neste trabalho a técnica de solução proposta para o problema de PSDEE, que é formulado como um modelo de programação não linear inteiro misto (PNLIM) é a metaheurística busca dispersa (BD). Trata-se de um método de busca evolucionária [10], que utiliza estratégias para a diversificação e intensificação semelhantes às da TS, o qual provou ser eficaz em uma variedade de problemas de otimização. Assim, este trabalho apresenta como diferencial, em relação a outros trabalhos de PSDEE disponíveis na literatura, a utilização da técnica BD para resolver problemas de PSDEE.

O sistema de codificação utilizado é a codificação sequencial aditiva proposta em [12]. A principal vantagem desta codificação é que toda proposta de solução configura redes radiais e facilita a combinação entre soluções durante o processo iterativo da busca dispersa. Para a seleção eficiente dos condutores a serem instalados em novas linhas e/ou recondutorados nas linhas existentes, utilizou-se uma

Este trabalho contou com o apoio financeiro da CAPES, FAPESP processo 13/23590-8 e CNPq processo 305371/2012-6.

S. G. B. d. Pádua, Universidade do Estado de Mato Grosso ” (UNEMAT), Tangará da Serra, Mato Grosso, Brasil, suzan@unemat.br

A. M. Cossi, Universidade Estadual Paulista "Júlio de Mesquita Filho" (UNESP), Ilha Solteira, São Paulo, Brasil, cossi@mat.feis.unesp.br

J. R. S. Mantovani, Universidade Estadual Paulista "Júlio de Mesquita Filho”(UNESP), Ilha Solteira, São Paulo, Brasil, mant@dee.feis.unesp.br heurística apresentada em [13]. Neste caso, uma escolha eficiente e não aleatória dos condutores, reduz o tempo computacional do algoritmo de $\mathrm{BD}$ e melhora o desempenho de redução da componente da função objetivo relativa às perdas do sistema.

A metodologia proposta foi aplicada em dois sistemas testes da literatura, um com 54 barras [5] e outro com 417 barras [14]. Foi utilizado o planejamento estático de curto prazo com um horizonte de planejamento de 5 anos considerando que os investimentos são realizados apenas no primeiro ano. Para os custos das perdas foram considerados 3 diferentes cenários de operação e seus valores atualizados para o valor presente, através de uma taxa de atualização.

O trabalho está organizado da seguinte forma: na Seção 2 apresenta-se em detalhes a formulação matemática do problema de PSDEE; na Seção 3 descreve-se o algoritmo Busca Dispersa desenvolvido para resolver o problema de PSDEE; na Seção 4 apresentam-se os resultados e comentários dos testes; e na Seção 5 as conclusões finais.

\section{Modelo MATEMÁTICO}

O modelo considerado para o PSDEE é não linear inteiro misto, em que a função objetivo consiste em minimizar os custos de expansão e operação da rede de distribuição de energia considerando um horizonte de planejamento preestabelecido. Este modelo é dado pelo seguinte conjunto equações:

$$
\begin{aligned}
& \text { Minimizar: } \\
& \begin{aligned}
F O= & \sum_{l \in \Omega_{S}} \sum_{k \in \Phi_{l}} C F S_{k l} y_{k l}+\sum_{i \in \Gamma} \sum_{a \in \Psi} \sum_{b \in \Psi} C F C_{a b} L_{i} y_{i a b}+ \\
& +\sum_{t=1}^{a p} \sum_{\pi \in \mathrm{Y}} \sum_{i \in \Gamma} \sum_{a \in \Psi} \sum_{b \in \Psi} 3 C_{k W h} H_{\pi} R_{b} J_{\pi i b}^{2} y_{i a b} L_{i}\left(\frac{1}{1+t j}\right)^{t}
\end{aligned}
\end{aligned}
$$

s. a.

$$
\begin{aligned}
& P_{s i}-P_{d i}-\sum_{j \in \Omega_{i}}\left(x_{i j} P_{i j}\right)=0, \quad \forall i \in \Omega \\
& Q_{s i}-Q_{d i}-\sum_{j \in \Omega_{i}}\left(x_{i j} Q_{i j}\right)=0, \quad \forall i \in \Omega \\
& P_{i j}=V_{i}^{2} g_{i j}-V_{i} V_{j}\left(g_{i j} \cos \theta_{i j}+b_{i j} \operatorname{sen} \theta_{i j}\right) \\
& Q_{i j}=V_{i}^{2} b_{i j}-V_{i} V_{j}\left(g_{i j} \operatorname{sen} \theta_{i j}-b_{i j} \cos \theta_{i j}\right) \\
& \left|f_{i b}^{C a l}\right| \leq f_{b}^{\max }, \quad i \in \Gamma \\
& \sum_{j \in \Omega_{l}} S_{j l}^{C}+\sum_{i \in \Gamma_{l}} S_{i l}^{L} \leq S_{k l}^{S} y_{k l}, \quad l \in \Omega_{s}, \quad k \in \Phi_{l} \\
& V^{\min } \leq V_{j} \leq V^{\max }, \quad j \in \Omega
\end{aligned}
$$




$$
\begin{aligned}
& \sum_{i \in \Omega_{l}} \sum_{a \in \Psi} \sum_{b \in \Psi} y_{i a b}=|\Omega|-\left|\Omega_{S}\right| \\
& R_{f} \leq R_{f}^{\max } \\
& \sum_{a \in \Psi} \sum_{b \in \Psi} y_{i a b} \leq 1, \quad \forall i \in \Gamma \\
& y_{k l}, \quad y_{i a b}, \quad x_{i j} \in\left\{\begin{array}{ll}
0, & 1
\end{array}\right\}
\end{aligned}
$$

Sendo:

ap: $\quad$ Total de anos do horizonte de planejamento;

$b_{i j}$ : $\quad$ Susceptância da linha $\mathrm{i}-\mathrm{j}$;

$C_{k w h}: \quad$ Custo do kWh;

$C F S_{k l}$ : Custo fixo da construção (ou repotencialização) da subestação do tipo $k$ na barra $l$;

Custo fixo pelo recondutoramento (ou instalação)

$C F C$. do condutor do tipo $a$ para o tipo $b$. Para o caso de instalação de novos condutores $a=0$ e o condutor instalado é do tipo $b$;

$f_{b}^{\max }$ : Fluxo máximo da corrente permitido no condutor

$f_{b} \quad$ do tipo $b$;

$f_{i b}^{C a l}$ : Fluxo da corrente calculado na linha $i$ com um

condutor do tipo $b$

$g_{i j}: \quad$ Condutância da linha $i-j$;

$H_{\pi}$ : Total de horas do ano correspondente ao cenário

de operação $\pi$;

$J_{\pi i b}$ : $\quad$ Corrente no condutor do tipo $b$ na linha $i$ no

cenário de operação $\pi$;

$L_{i}: \quad$ Comprimento da linha $i$;

$P_{d i}$ : $\quad$ Potência ativa demandada na barra $i$;

$P_{i j}: \quad \quad \quad \quad$ Fluxo de potência ativa na linha $i-j$;

$P_{s i}$ : $\quad$ Potência ativa gerada na barra $i$;

$Q_{d i}$ : Potência reativa demandada na barra $i$;

$Q_{i j}$ : $\quad$ Fluxo de potência reativa na linha $i-j$;

$Q_{s i}$ : $\quad$ Potência reativa gerada na barra $i$;

$R_{b}$ : Resistência do condutor do tipo $b$;

$R_{f}$ : $\quad$ Custos dos novos investimentos;

$R_{f}^{\max }: \quad$ Recurso financeiro máximo disponível para novos

investimentos;

$S_{i l}^{L}$ : $\quad$ Perdas na linha $i$ conectada à subestação da barra $l$

$S_{i l}^{L}$ no cenário de operação com carga pesada;

$S_{j l}^{C}$ : Potência consumida na barra $j$ conectada à

subestação da barra $l$;

$S_{k l}^{S}$ : $\quad$ Capacidade da subestação do tipo $k$ na barra $l$;

$T C_{i}$ : $\quad$ Tipo do condutor instalado na linha $i$;

tj : $\quad$ Taxa de juros;

$T S_{l}$ : $\quad$ Tipo de subestação inicial na barra $l$;

$V_{(.)}$: $\quad$ Magnitude de tensão na barra (.);

$V^{\max }$ Limite máximo para à magnitude de tensão nas barras do sistema;

$V^{\text {min }}: \quad$ Limite mínimo para à magnitude de tensão nas barras do sistema; $x_{i j}: \quad \begin{aligned} & \text { Variável binária que determina se o circuito entre } \\ & \text { as barras } i \text { e } j \text { está fechado; }\end{aligned}$

Variável binária de decisão para o recondutoramento (ou instalação) do condutor do

$y_{i a b}: \quad$ tipo $a$ para o tipo $b$ na linha $i$. Para o caso de instalação de novos condutores $a=0$ e o condutor instalado é do tipo $b$;

Variável binária de decisão para a construção (ou

$y_{k l}$ : repotencialização) da subestação do tipo $k$ na barra $l$;

$\Gamma_{l}$ : Conjunto de linhas conectadas à subestação $l$, $\Gamma_{l}: \quad \operatorname{com} l \in \Omega_{s}$

$\Gamma: \quad$ Conjunto de linhas propostas ou existentes;

$\Omega$ : $\quad$ Conjunto de barras propostas ou existentes na rede;

$\Omega_{S}$ : Conjuntos de barras com subestações propostas ou existentes;

$\Omega_{i}: \quad$ Conjunto de barras conectadas à barra $i$;

$\Omega_{l}$ : Conjunto de barras conectadas à subestação $l$,

$\operatorname{com} l \in \Omega_{s}$

$\Psi$ : $\quad$ Conjunto dos tipos de condutores disponíveis;

$\Phi_{l}$ : Conjunto dos tipos de subestações disponíveis para à barra $l$, com $l \in \Omega_{s}$;

$\theta_{i j}:$

Diferença angular entre as tensões das barras $i-j$;

Y : Conjunto dos cenários de operação (carga leve, normal e pesada);

De acordo com a função objetivo (1), os custos com a expansão (custos fixos) referem-se à soma dos investimentos com construção e/ou repotencialização de subestações e instalação e/ou recondutoramento de linhas; e os custos com a operação da rede (custos variados) referem-se às perdas técnicas de operação da rede durante o período de planejamento. As restrições físicas, financeiras e operacionais do serviço de fornecimento de energia elétrica a considerar são: suprimento das demandas de potências ativa e reativa para cada barra do sistema de distribuição (2) e (3), balanço de potência entre as barras (4) e (5), limites nas capacidades de correntes das linhas (6), limite de capacidade das subestações (7), limites máximo e mínimo da magnitude das tensões nos pontos de consumo (8), as restrições (2), (3) e (9) garantem a radialidade do sistema projetado, disponibilidade de recursos financeiros para novos investimentos (10), escolha de apenas um tipo de condutor para as linhas novas ou recondutoradas (11).

\section{TÉCNICA DE SOLUÇÃO}

A proposta de solução do problema de planejamento é a BD, adaptada para solução de problemas de Programação não Linear Inteiro Misto.

As diversas implementações da metaheurística BD são basicamente formadas por cinco etapas fundamentais, e o que difere entre uma implementação simples ou uma mais avançada é a incorporação de estratégias em cada uma dessas etapas visando um melhor desempenho do método. Essas estratégias definem, por exemplo: a maneira como será realizada e quais características das soluções serão priorizadas 
para a construção e/ou atualização dos conjuntos utilizados na BD; se algum tipo de memória será incorporado no algoritmo; quantas e como as soluções serão combinadas para a construção de uma nova solução; e os critérios de convergência adotados. Considerando um problema de otimização do tipo:

$$
\left\{\begin{array}{c}
\operatorname{Min} f(x) \\
s . a . \\
g(x) \leq 0 \\
h(x)=0 \\
x^{\min } \leq x \leq x^{\max }
\end{array}\right.
$$

As cinco etapas fundamentais para solução deste problema são:

- Etapa 1 - geração de soluções com diversificação: usada para construir um conjunto $P$ composto por PSize soluções diversificadas que são a base do algoritmo;

- Etapa 2 - melhoria local de soluções: transforma uma solução visando torná-la factível ou melhorando o valor da função objetivo;

- Etapa 3 - atualização das soluções do conjunto de referência (RefSet): empregada para a construção e manutenção do conjunto RefSet. Construção seleciona $b$ soluções distintas do conjunto $P$, conforme uma regra preestabelecida, visando incluir alta qualidade (em relação à função objetivo) e diversidade no conjunto RefSet. Manutenção realizada a atualização das soluções do conjunto RefSet sempre quando uma nova solução encontrada cumpre os requisitos para ingressar no RefSet. Assim a dimensão do RefSet será sempre constante, mas a qualidade das soluções vão melhorando durante o processo de busca. Geralmente, a atualização do RefSet se realiza unicamente por qualidade. Após terse realizado todas as combinações possíveis das soluções (atualização estática), as novas soluções encontradas durante as etapas de combinação e melhoria local são armazenadas no conjunto Pool para só depois serem avaliadas durante a atualização do RefSet.

- Etapa 4 - geração de subconjuntos de soluções: gera $s$ diferentes subconjuntos de soluções com as soluções de referência que são usados na etapa de combinação de soluções. Para isso, esses $s$ subconjuntos são armazenados no conjunto NovoSubconjuntos, e só depois que todos os subconjuntos forem criados é que a etapa de combinação de soluções se inicia.

- Etapa 5 - combinação de soluções: Usa os $s$ subconjuntos gerados e armazenados no conjunto NovoSubconjuntos através da etapa de geração de subconjuntos. Com o objetivo de encontrar novas soluções, denominadas soluções testes, realizam-se combinações entre as soluções do primeiro subconjunto $s$ e após todas as combinações serem realizadas esse subconjunto é retirado do conjunto NovoSubconjuntos e o processo é repetido com o próximo subconjunto até que todos os subconjuntos $s$ sejam utilizados e retirados do conjunto NovoSubconjuntos, ou seja, NovoSubconjuntos $=\varnothing$. O método de combinação das soluções deve levar em conta a estrutura física do problema real e a maneira como essas soluções são codificadas. Aos serem criadas, as soluções teste passam pela etapa de melhoria local e são armazenadas no conjunto Pool usado durante a etapa de atualização do RefSet.

Considerando o problema de planejamento (1)-(12), as diferentes etapas da BD aplicadas na solução do modelo de otimização geral (13) foram adaptadas à sua solução. Inicialmente foi escolhido um sistema de codificação adequado para implementação da BD, adotando-se a codificação sequencial aditiva [12], que inicia com todas as barras de cargas (existentes e futuras) isoladas do sistema e prossegue, conectando uma barra por vez, até que todas as barras estejam conectadas à rede de distribuição. Essa codificação é representada pelo vetor $v$ ilustrado na Figura 1:

$$
v=\begin{array}{|l|l|l|l|l|}
\hline v_{1} & v_{2} & v_{3} & \cdots & v_{m} \\
\hline
\end{array}
$$

Figura 1. Vetor de codificação sequencial aditiva.

sendo $m$ o número de barras de cargas existentes e futuras na rede de distribuição e $v_{i} \in[0,1[$, que após serem decodificados definirão as linhas da rede responsáveis pela conexão das barras de carga [12].

A codificação sequencial aditiva reduz bastante o espaço de busca, pois só gera configurações radiais. Além disso, os operadores usados nas etapas de combinação e melhoria local da $\mathrm{BD}$ podem ser implementados da forma convencional, como a combinação de um ponto que consiste em trocar parcelas de duas soluções escolhidas e a melhoria local 2-opt que troca dois elementos de uma mesma solução de lugar, garantindo sempre a radialidade nas soluções resultantes.

$\mathrm{Na}$ Figura 2 apresenta-se o diagrama de blocos do algoritmo de $\mathrm{BD}$ implementado neste trabalho. Este diagrama foi desenvolvido para o problema genérico (13).

\section{A. Estratégia para a geração de soluções com diversidade:}

Um elemento importante da BD é a técnica utilizada para a geração de soluções diversificadas que compõem o conjunto $P$. O ideal é o uso de um procedimento sistemático que consiga gerar soluções que difiram significativamente umas das outras [10]. A estratégia adotada, neste trabalho, para geração de soluções com diversidade foi apresentada por [11] e emprega randomização controlada e frequência baseada em memória. Para gerar o vetor $v$ da codificação sequencial aditiva (Figura 1), o intervalo [0, 1[ é dividido em 4 intervalos menores de tamanhos iguais e cada intervalo é representado por $t_{j}$ para $j=1, \ldots, 4$. O elemento $v_{i}$ para $i=1, \ldots, m$, é construído em duas etapas. $\mathrm{Na}$ primeira etapa seleciona-se randomicamente um dos $t_{j}$ intervalos. A probabilidade de selecionar cada intervalo $t_{j}$ é inversamente proporcional à quantidade de vezes que esse intervalo já foi selecionado em iterações anteriores durante o processo de construção do conjunto $P$. Sendo assim, na primeira iteração todos os $t_{j}$ 
intervalos têm a mesma chance de serem escolhidos. Para as iterações $k>1$ a frequência de vezes que cada intervalo já foi selecionado em iterações anteriores $\left(\right.$ freq $\left.t_{i j_{k-1}}\right)$ para compor o elemento $v_{i}$ é armazenada e a probabilidade do intervalo $t_{j}$ ser selecionado é definida pela equação (14).

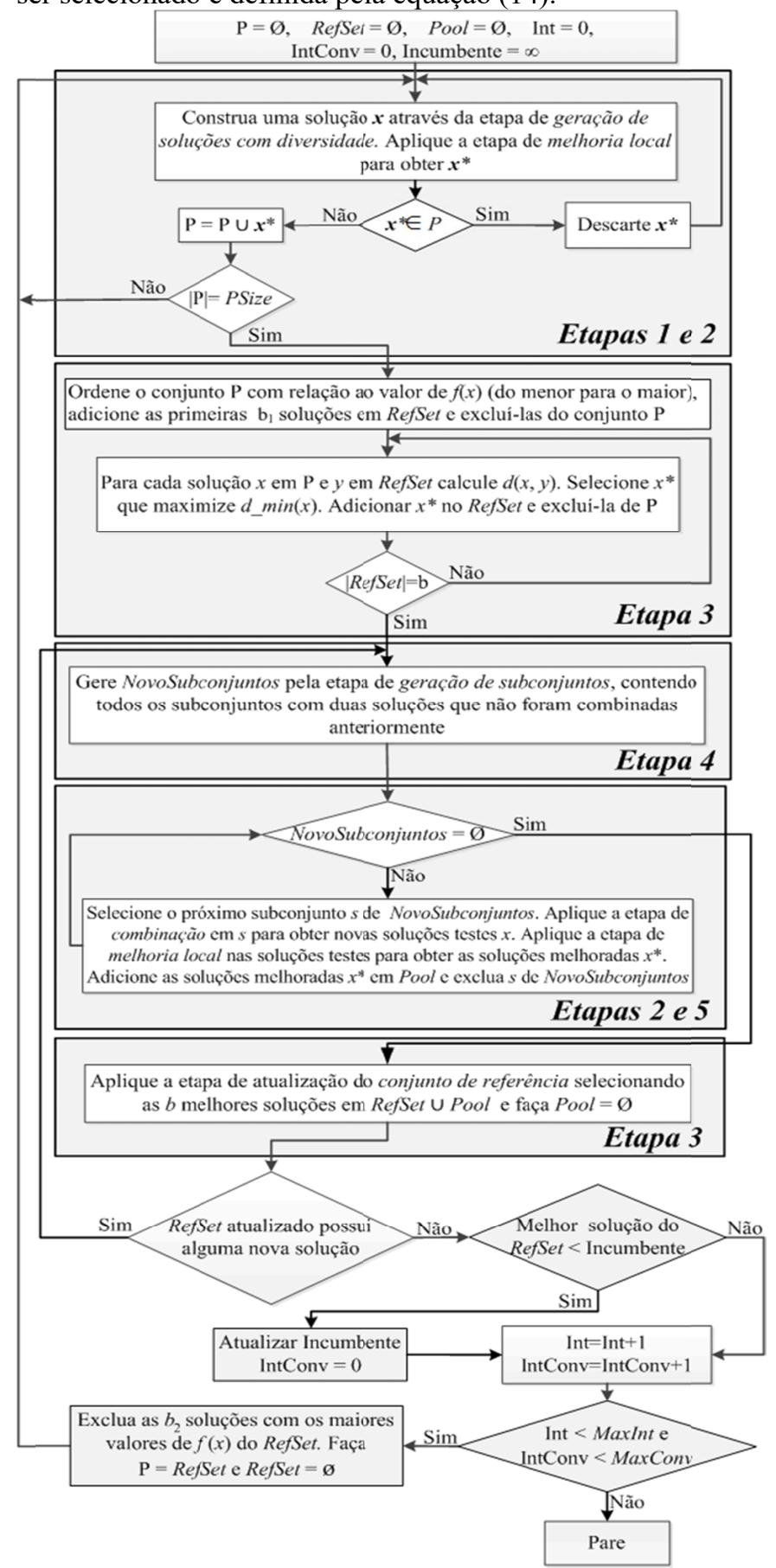

Figura 2. Diagrama de blocos do algoritmo BD implementado.

$$
\text { Prob } t_{i j_{k}}=\frac{(k-1)-\text { freq } t_{i j_{k-1}}}{\sum_{j=1}^{4}\left[(k-1)-\text { freq } t_{i j_{k-1}}\right]}
$$

Na segunda etapa, a variável $v_{i}$ é randomicamente gerada dentro do intervalo selecionado durante a primeira etapa. $\mathrm{O}$ processo é repetido até que o vetor $v$ esteja completo. Em seguida, essa solução é decodificada.

\section{B. Estratégias de Melhorias das soluções no PSDEE}

Quando a escolha dos condutores a serem instalados nas linhas novas e/ou recondutorados nas linhas existentes é feita de forma eficiente, e não aleatória, aumenta a eficiência do algoritmo BD. Para a seleção eficiente dos condutores é aplicada a heurística apresentada em [13], que garante a escolha de apenas um condutor para cada nova instalação ou recondutoramento (restrição (11)). Essa heurística é dividida em duas fases. Na primeira fase a escolha do condutor é feita visando o menor custo e atender a restrição de corrente (6). $\mathrm{Na}$ segunda fase, se existirem barras com tensão abaixo da tensão mínima (8) as bitolas dos condutores a jusante são aumentadas de acordo com um índice de sensibilidade.

As restrições (2) e (3) relacionadas com o atendimento das demandas de potências ativa e reativa para cada barra é considerada através da solução de um algoritmo de fluxo de potência de varredura convencional [15].

A codificação sequencial aditiva garante a radialidade do sistema (restrições (2), (3) e (11)), porém, não consegue controlar a quantidade de cargas conectadas em cada subestação e mesmo com a escolha eficiente dos condutores pode obter soluções que violem as restrições (6), (7) e (8). Essas infactibilidades são penalizadas na função de adaptação para tornar tais soluções não atraentes durante a busca. A função de adaptação avalia cada configuração candidata a solução do problema de PSDEE, e é formada pela função objetivo mais o termo de penalização das restrições violadas, dada por:

$$
F O_{p}=F O+\sum_{i \in C R} \mu_{i}\left|R_{i}\right|
$$

sendo $\mu_{i}$ e $\left|R_{i}\right|$, respectivamente, o fator de penalidade e o grau de violação da restrição $i$ e $C R$ o conjunto contendo as restrições (6), (7) e (8).

Assim, a etapa de melhoria local não visa diretamente resolver problemas de infactibilidade, apenas realiza uma busca na vizinhança da solução $v_{d}$ (solução decodificada) através dos vetores $v^{*}$ e $v^{* *}$, com o intuito de aumentar a qualidade da solução com relação ao valor da função objetivo, ou pode realizar uma mudança total na solução $v_{d}$ através do vetor $v^{* * *}$, dados por:

$v_{i}^{*}=\left\{\begin{array}{cc}v_{i} & i=1, \ldots, m-k \\ r_{i}^{*}\left(1-v_{i}\right) & i=m-k+1, \ldots, m\end{array}\right.$

sendo $k=\operatorname{int}(80 \% . m), m$ é a dimensão do vetor $v$ e $r_{i}^{*} \in[0,1[$ é gerado randomicamente.

$v_{i}^{* *}=\left\{\begin{array}{cc}v_{i} & i=1, \ldots, m-k \\ v_{2 m-k-1-i} & i=m-k+1, \ldots, m\end{array}\right.$

sendo $k=r^{* *} \operatorname{int}(25 \% . m)+4$, e $r_{i}^{* *} \in[0,1]$. 
$v_{i}^{* * *}=\left\{\begin{array}{cc}v_{k-i+1} & i=1, \ldots, k \\ v_{i} & i=k+1, \ldots, m\end{array}\right.$

sendo $k=r^{* * *} \operatorname{int}(25 \% . m)+4$, e $r_{i}^{* * *} \in[0,1]$.

A solução melhorada é a que possui o menor valor da função objetivo entre a $v, v^{*}, v^{* *}$ e $v^{* * *}$.

\section{Construção e atualização do conjunto de referência}

A seleção das $b$ soluções de referência que compõem o conjunto RefSet inicial depende da qualidade e da diversidade, dadas por:

- Seleção por qualidade: ordenar o conjunto $P$ de acordo com o valor da função objetivo. As $b_{1}$ (com $\left.b_{1}<b\right)$ primeiras soluções de qualidade são selecionadas para compor o RefSet e retiradas do conjunto $P$.

- Seleção por diversidade: O cálculo da dissimilaridade entre as soluções $x$ e $y$ leva em consideração a quantidade de linhas distintas $\left(d_{x y}\right)$, o módulo da diferença do valor da função objetivo e o número de barras alimentadas pela subestação $i$, sendo $n$ o total de subestações.

$$
\begin{aligned}
d(x, y)= & \alpha \cdot d_{x y}+\beta|F O(x)-F O(y)| \\
& +\delta \sum_{i=1}^{n}\left|n b_{i x}-n b_{i y}\right|
\end{aligned}
$$

sendo $\alpha, \beta$ e $\delta \in \mathrm{R}$ definidos a priori. Como critério de escolha das soluções tem-se a regra de distância mínimamáxima. São calculadas as distâncias entre as soluções $x$ que fazem parte do conjunto $P$ com as soluções $y$ que já estão no conjunto RefSet. Para cada solução $x \in P$ é escolhida a distância mínima entre ela e as soluções $y \in \operatorname{RefSet}$, ou seja:

$$
d_{\text {min }}(x)=\operatorname{Min}_{y \in \operatorname{RefSet}}\{d(x, y)\}
$$

Assim, seleciona-se a solução $x$ com a máxima distância, ou seja, a solução que possua os maiores valores de $d_{\min }(x)$ e ela é incluída no RefSet. O processo é repetido até que o conjunto RefSet contenha $b$ soluções, para isso serão selecionadas $b_{2}$ soluções, sendo $b_{2}=b-b_{1}$.

Segundo [11] o ideal, ao construir o RefSet inicial, é que a quantidade de soluções escolhidas por qualidade e por diversidade sejam iguais, por outro lado, durante a atualização do RefSet a qualidade das soluções é mais importante que a diversidade. Assim, a atualização é efetuada visando apenas a qualidade.

\section{Estratégias de geração de subconjuntos e combinação de soluções de referência}

Na etapa de geração de subconjuntos para a solução do problema de PSDEE criam-se todos os subconjuntos $s$ contendo duas soluções do RefSet, sendo que pelo menos uma solução seja "nova", ou seja, pelo menos uma solução tenha acabado de ingressar no conjunto RefSet e por isso ainda não foi combinada em iterações anteriores garantindo que as soluções já combinadas anteriormente não foram parte do mesmo subconjunto $s$. Esses subconjuntos $s$ são armazenados no conjunto NovoSubconjuntos para serem usados na etapa de combinação de soluções que só inicia depois que todos os subconjuntos $s$ forem criados. As soluções $v^{\prime}$ e $v^{\prime \prime}$ de cada subconjunto $s$ são combinadas para criar três novas soluções denominadas soluções testes, dadas pelas equações (21), (22) e (23) que são melhoradas e armazenadas no conjunto Pool e o subconjunto $s$ é retirado do conjunto NovoSubconjuntos. A etapa termina quando foram realizadas as combinações em todos os subconjuntos $s$, ou seja, NovoSubconjuntos $=\varnothing$.

$v_{i}^{1}=\left\{\begin{array}{cc}\frac{v_{i}^{\prime} \cdot F\left(v^{\prime}\right)+v^{\prime \prime}{ }_{i} \cdot F\left(v^{\prime \prime}\right)}{F\left(v^{\prime}\right)+F\left(v^{\prime \prime}\right)} & \text { se } v_{i}^{\prime} \text { ou } v^{\prime \prime}{ }_{i} \neq 0 \\ 0,99 & \text { se } v_{i}^{\prime} \text { e } v^{\prime \prime}{ }_{i}=0\end{array}\right.$

para $i=1, \ldots, m$.

$v_{i}^{2}=\left\{\begin{array}{cc}v^{\prime}{ }_{i} & \text { para } i=1, \ldots, k \\ v^{\prime \prime}{ }_{i} & \text { para } i=k+1, \ldots, m\end{array}\right.$

$v_{i}^{3}=\left\{\begin{array}{l}{v^{\prime \prime}}_{i} \quad \text { para } i=1, \ldots, k^{*} \\ v^{\prime}{ }_{i} \quad \text { para } i=k^{*}+1, \ldots, m\end{array}\right.$

sendo $k$ e $k^{*} \in N$, escolhidos randomicamente no intervalo de ] $1, m[$.

\section{E. Critérios de convergência da $B D$}

O algoritmo $\mathrm{BD}$ é considerado convergido quando é atingido o número máximo de iterações preestabelecido IntMax ou quando a solução incumbente (melhor valor da FO encontrado) não melhorar durante um número preestabelecido de iterações consecutivas, MaxConv.

\section{Testes E REsultados}

A metodologia proposta foi aplicada em dois sistemas teste da literatura, um com 54 barras e outro com 417 barras. Nos testes, considera-se cada sistema é equilibrado e pode ser representado apenas pelo diagrama unifilar de componente de sequência positiva. O planejamento realizado é o estático de curto prazo. Nas Tabelas I e II apresentam-se os parâmetros necessários para o modelo de PSDEE definido pelas equações (1) - (12).

Os custos com a expansão da rede são considerados no início do horizonte de planejamento e os custos com as perdas técnicas, nos diferentes cenários de operação, são atualizados para o valor presente, através de uma taxa de atualização.

TABELA I. PARÂMETROS PARA O MODELO DE PSDEE.

\begin{tabular}{ccccc}
\hline $\begin{array}{c}V^{\max } \\
(\mathrm{pu})\end{array}$ & $\begin{array}{c}V^{\operatorname{mim}} \\
(\mathrm{pu})\end{array}$ & $\begin{array}{c}C_{k W h} \\
(\mathrm{US} \$ / \mathrm{kWh})\end{array}$ & $\begin{array}{c}t j \\
(\mathrm{aa})\end{array}$ & $\begin{array}{c}a p \\
(\text { anos })\end{array}$ \\
\hline 1,05 & 0,95 & 0,10 & 0,10 & 5 \\
\hline
\end{tabular}


TABELA II. PARÂMETROS PARA OS CENÁRIOS DE OPERAÇÃo DO PSDEE.

\begin{tabular}{c|cc}
\hline $\begin{array}{c}\text { Cenário de } \\
\text { operação }(\pi)\end{array}$ & $\begin{array}{c}H_{\pi} \\
\text { (horas) }\end{array}$ & $\begin{array}{c}\text { Fator de } \\
\text { carregamento }\end{array}$ \\
\hline 1 & 1000 & 0,30 \\
2 & 6760 & 0,50 \\
3 & 1000 & 1,00 \\
\hline
\end{tabular}

\section{A. Sistema de 54 barras}

Trata-se de um sistema de distribuição de $15 \mathrm{kV}$, com 54 barras, sendo duas subestações existentes que permitem ser repotencializadas e duas subestações candidatas [5]. O sistema inicial possui 16 barras energizadas e 34 barras que deverão ser alimentadas durante o período de planejamento. A Figura 3 ilustra o sistema inicial em operação e a Figura 4 a topologia otimizada do sistema obtida através da BD. Para a instalação dos novos alimentadores ou recondutoramento dos alimentadores já existentes tem-se disponível 6 diferentes tipos de condutores, dados na Tabela III. Na Tabela IV apresentam-se os custos para recondutoramento e/ou instalação de novos condutores. De acordo com a Tabela IV, no caso de um recondutoramento haveria a possibilidade de substituição de um condutor do Tipo A (Tipo $A \neq 0$ ) para outro condutor de maior capacidade do Tipo B, e no caso de instalação de novos condutores no sistema (Tipo $\mathrm{A}=0$ ), seriam escolhidos condutores do Tipo B. Os dados completos do sistema encontram-se nas Tabelas V - VII.

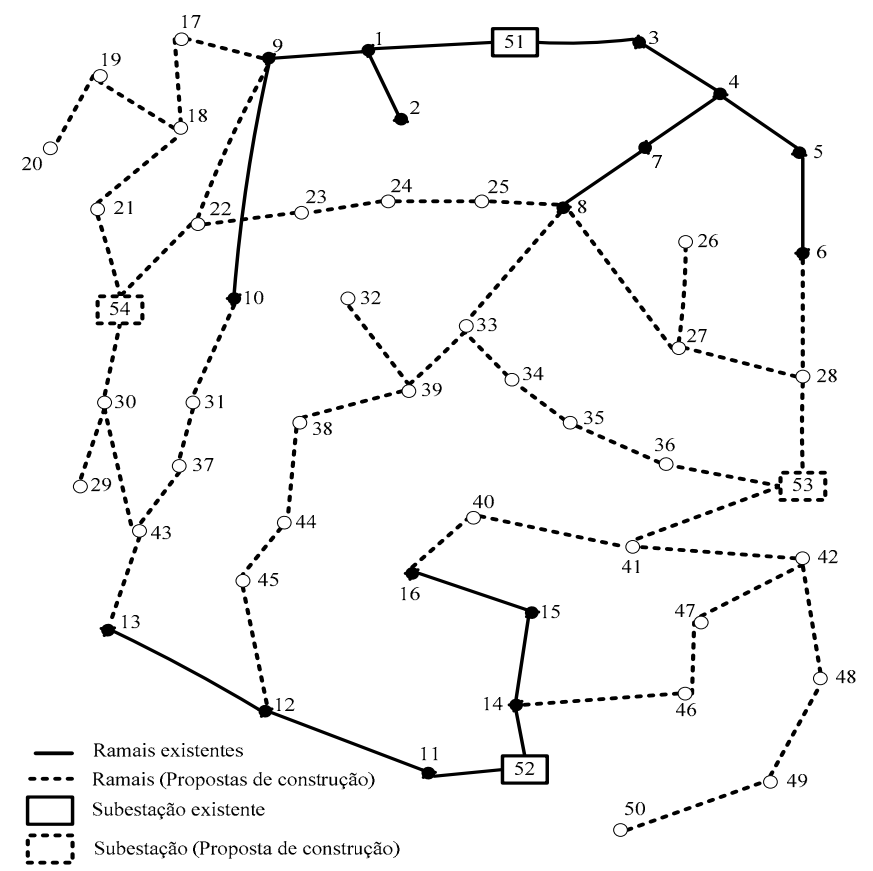

Figura 3. Configuração inicial do sistema de 54 barras.
TABELA III. DADOS DOS CONDUTORES UTILIZADOS PARA O SISTEMA DE 54 BARRAS.

\begin{tabular}{cccc}
\hline $\begin{array}{c}\text { Tipo } \\
\text { Condutor }\end{array}$ & $\begin{array}{c}I_{b}^{\max } \\
(\mathrm{A})\end{array}$ & $\begin{array}{c}R_{b} \\
(\Omega / \mathrm{Km})\end{array}$ & $\begin{array}{c}X_{b} \\
(\Omega / \mathrm{Km})\end{array}$ \\
\hline 1 & 150 & 0,3655 & 0,2520 \\
2 & 250 & 0,2359 & 0,2402 \\
3 & 350 & 0,1827 & 0,1260 \\
4 & 400 & 0,1460 & 0,1233 \\
5 & 500 & 0,1180 & 0,1201 \\
6 & 600 & 0,0966 & 0,1140 \\
\hline
\end{tabular}

TABELA IV. CUSTOS PARA RECONDUTORAMENTO E/OU INSTALAÇÃO PARA OS CONDUTORES DA TABELA III.

\begin{tabular}{c|cccccc}
\hline \multicolumn{7}{c}{$C F C_{A B}\left(10^{3} U S \$ / K m\right)$} \\
Tipo A & 1 & 2 & 3 & 4 & 5 & 6 \\
\hline 0 & 20 & 30 & 42 & 50 & 67 & 85 \\
1 & - & 23 & 37 & 40 & 58 & 76 \\
2 & - & - & 30 & 35 & 50 & 66 \\
3 & - & - & - & 29 & 43 & 53 \\
4 & - & - & - & - & 35 & 45 \\
5 & - & - & - & - & - & 38 \\
\hline
\end{tabular}

TABELA V. CAPACIDADE E CUSTOS DAs SUBESTAÇÕES.

\begin{tabular}{cccccc}
\hline$l$ & $T S_{l}$ & $\begin{array}{c}S_{1 l}^{S} \\
(\mathrm{MVA})\end{array}$ & $\begin{array}{c}S_{2 l}^{S} \\
(\mathrm{MVA})\end{array}$ & $\begin{array}{c}C F S_{1 l} \\
\left(10^{3} \mathrm{US} \$\right)\end{array}$ & $\begin{array}{c}C F S_{2 l} \\
\left(10^{3} \mathrm{US} \$\right)\end{array}$ \\
\hline 51 & 1 & 16,7 & 33,4 & - & 1400 \\
52 & 1 & 16,7 & 30 & - & 1200 \\
53 & 0 & 22 & - & 3000 & - \\
54 & 0 & 22 & - & 3000 & - \\
\hline
\end{tabular}

TABELA VI. DADOS DE BARRA DO SISTEMA DE 54 BARRAS.

\begin{tabular}{ccc|ccc|ccc}
\hline$l$ & $\begin{array}{c}P_{l} \\
(k W)\end{array}$ & $\begin{array}{c}Q_{l} \\
(k v a r)\end{array}$ & $l$ & $\begin{array}{c}P_{l} \\
(k W)\end{array}$ & $\begin{array}{c}Q_{l} \\
(k v a r)\end{array}$ & $l$ & $\begin{array}{c}P_{l} \\
(k W)\end{array}$ & $\begin{array}{c}Q_{l} \\
(k v a r)\end{array}$ \\
\hline 1 & 3780 & 1830.7 & 19 & 1260 & 610.2 & 37 & 1890 & 915.4 \\
2 & 1350 & 653.8 & 20 & 720 & 348.7 & 38 & 990 & 479.5 \\
3 & 630 & 305.1 & 21 & 1620 & 784.6 & 39 & 900 & 435.9 \\
4 & 990 & 479.5 & 22 & 990 & 479.5 & 40 & 1260 & 610.2 \\
5 & 2340 & 1133.3 & 23 & 900 & 435.9 & 41 & 810 & 392.3 \\
6 & 630 & 305.1 & 24 & 450 & 217.9 & 42 & 1080 & 523.1 \\
7 & 900 & 435.9 & 25 & 810 & 392.3 & 43 & 1170 & 566.7 \\
8 & 1710 & 828.2 & 26 & 1080 & 523.1 & 44 & 1260 & 610.2 \\
9 & 1080 & 523.1 & 27 & 1350 & 653.8 & 45 & 720 & 348.7 \\
10 & 2610 & 1264.1 & 28 & 630 & 305.1 & 46 & 1620 & 784.6 \\
11 & 270 & 130.8 & 29 & 1260 & 610.2 & 47 & 900 & 435.9 \\
12 & 1620 & 784.6 & 30 & 2340 & 1133.3 & 48 & 720 & 348.7 \\
13 & 990 & 479.5 & 31 & 630 & 305.1 & 49 & 450 & 217.9 \\
14 & 900 & 435.9 & 32 & 1530 & 741 & 50 & 720 & 348.7 \\
15 & 1260 & 610.2 & 33 & 2610 & 1264.1 & 51 & 0 & 0 \\
16 & 1710 & 828.2 & 34 & 1080 & 523.1 & 52 & 0 & 0 \\
17 & 630 & 305.1 & 35 & 810 & 392.3 & 53 & 0 & 0 \\
18 & 1080 & 523.1 & 36 & 270 & 130.8 & 54 & 0 & 0 \\
\hline
\end{tabular}

TABELA VII. DADOS DE LINHA DO SISTEMA DE 54 BARRAS.

\begin{tabular}{ccc|ccc|ccc}
\hline$i$ & $L_{i}(\mathrm{~km})$ & $T C_{i}$ & $i$ & $L_{i}(\mathrm{~km})$ & $T C_{i}$ & $i$ & $L_{i}(\mathrm{~km})$ & $T C_{i}$ \\
\hline 1 & 0.562 & 2 & 22 & 0.500 & 0 & 43 & 0.686 & 0 \\
2 & 0.436 & 2 & 23 & 0.750 & 0 & 44 & 0.812 & 0 \\
3 & 0.624 & 2 & 24 & 0.936 & 0 & 45 & 0.562 & 0 \\
4 & 0.500 & 2 & 25 & 0.686 & 0 & 46 & 0.936 & 0 \\
5 & 0.624 & 2 & 26 & 0.562 & 0 & 47 & 0.374 & 0 \\
6 & 0.624 & 2 & 27 & 0.436 & 0 & 48 & 0.436 & 0 \\
7 & 0.500 & 2 & 28 & 0.562 & 0 & 49 & 0.436 & 0 \\
\hline
\end{tabular}




\begin{tabular}{ccc|ccc|ccc}
\hline 8 & 0.686 & 2 & 29 & 0.750 & 0 & 50 & 0.500 & 0 \\
9 & 0.624 & 2 & 30 & 0.686 & 0 & 51 & 0.624 & 0 \\
10 & 1.436 & 2 & 31 & 0.624 & 0 & 52 & 0.624 & 0 \\
11 & 0.750 & 2 & 32 & 1.000 & 0 & 53 & 0.750 & 0 \\
12 & 0.750 & 2 & 33 & 0.562 & 0 & 54 & 0.500 & 0 \\
13 & 0.562 & 2 & 34 & 0.624 & 0 & 55 & 0.750 & 0 \\
14 & 0.562 & 2 & 35 & 0.812 & 0 & 56 & 0.500 & 0 \\
15 & 0.624 & 2 & 36 & 0.500 & 0 & 57 & 0.750 & 0 \\
16 & 0.874 & 2 & 37 & 0.374 & 0 & 58 & 0.436 & 0 \\
17 & 0.624 & 0 & 38 & 0.624 & 0 & 59 & 0.624 & 0 \\
18 & 0.500 & 0 & 39 & 0.750 & 0 & 60 & 0.624 & 0 \\
19 & 0.812 & 0 & 40 & 0.500 & 0 & 61 & 0.686 & 0 \\
20 & 0.860 & 0 & 41 & 0.436 & 0 & & & \\
21 & 0.624 & 0 & 42 & 0.624 & 0 & & & \\
\hline
\end{tabular}

A função de adaptação (15) tem como principal objetivo tornar as soluções que violam alguma das restrições do problema de PSDEE não atraentes durante a busca. A escolha adequada das constantes de penalidade das restrições é de fundamental importância, afinal, o valor da função de adaptação é utilizado para o cálculo de dissimilaridade entre duas soluções (equação (19)) responsável pela escolha das soluções por diversidade que compõem o RefSet inicial. A Tabela VIII apresenta os parâmetros para o cálculo da dissimilaridade e os valores das constantes de penalidade das restrições utilizados para esse sistema. Esses valores foram definidos através de testes exaustivos.

TABela VIII. PARÂMETRos NECESSÁRIos NA BUSCA Dispersa.

\begin{tabular}{cccccc}
\hline$\mu_{6}$ & $\mu_{7}$ & $\mu_{8}$ & $\alpha$ & $\beta$ & $\delta$ \\
\hline 18168 & 2600 & 0 & 1 & $2,5 \cdot 10^{-7}$ & $1 / 3$ \\
\hline
\end{tabular}

Um fator crítico da $\mathrm{BD}$ é o ajuste dos parâmetros dimensionais dos conjuntos que compõem a base do algoritmo, ou seja:

- $\quad P$ Size: dimensão do conjunto $P$;

- $\quad b$ : dimensão do conjunto RefSet;

- $\quad b_{1}$ : quantidade de soluções de qualidade selecionadas para o RefSet inicial.

As Tabelas IX e $\mathrm{X}$ apresentam os resultados obtidos em testes realizados para diferentes valores desses parâmetros como método de avaliação da influência dos mesmos na convergência do método. Na Tabela IX são adotado como parâmetros para os critérios de convergência do algoritmo (MaxInt e MaxConv) os valores 100 e 50, respectivamente. Já na Tabela $X$ esses valores foram alterados para 200 e 75 , respectivamente. A BD é executada 20 vezes $(n)$ em cada caso, e para a sintetização e avaliação dos resultados obtidos são utilizadas as medidas estatísticas de dispersão dadas pelo valor médio da função objetivo $\overline{F O}$, o desvio padrão amostral $S$ e o coeficiente de variação $C V$, sendo:

$$
\begin{gathered}
S=\sqrt{\frac{\sum_{i=1}^{n}\left(F O_{i}-\overline{F O}\right)^{2}}{n-1}} \\
C V=\frac{S}{\overline{F O}} \cdot 100
\end{gathered}
$$

Adicionalmente é apresentado o tempo médio de execução $\bar{t}$ do algoritmo.

TABELA IX. BD PARA INTMAX $=100 \mathrm{E} M A X C O N V=50$.

\begin{tabular}{ccccccc}
\hline PSize & $b$ & $b_{1}$ & $\overline{F O}$ (US\$) & $S$ & $C V(\%)$ & $\bar{t}(\mathrm{~s})$ \\
\hline \multirow{3}{*}{100} & 10 & 5 & 7069152 & 2885 & 0.040 & 156 \\
& 12 & 6 & 7068585 & 2395 & 0.033 & 170 \\
& 14 & 7 & 7067807 & 0 & 0 & 287 \\
\hline \multirow{3}{*}{150} & 10 & 5 & 7070430 & 3809 & 0.053 & 150 \\
& 12 & 6 & 7068585 & 2395 & 0.033 & 197 \\
& 14 & 7 & 7069364 & 3194 & 0.045 & 275 \\
\hline
\end{tabular}

TABELA X. BD PARA INTMAX $=200 \mathrm{E} M A X C O N V=75$.

\begin{tabular}{ccccccc}
\hline PSize & $b$ & $b_{1}$ & $\overline{F O}$ (US\$) & $S$ & $C V(\%)$ & $\bar{t}(\mathrm{~s})$ \\
\hline \multirow{3}{*}{150} & 10 & 5 & 7068974 & 2851 & 0.040 & 258 \\
& 12 & 6 & 7068196 & 1740 & 0.024 & 241 \\
& 14 & 7 & 7068228 & 1882 & 0.026 & 399 \\
\hline \multirow{3}{*}{100} & 10 & 5 & 7068975 & 2851 & 0.040 & 205 \\
& 12 & 6 & 7068374 & 1875 & 0.026 & 330 \\
& 14 & 7 & 7068795 & 2560 & 0.036 & 532 \\
\hline
\end{tabular}

Os resultados das Tabelas IX e $\mathrm{X}$ indicam que tanto para os critérios de convergência IntMax $=100$ e MaxConv $=50$ como para IntMax $=200$ e MaxConv $=75$ a BD apresentou um melhor desempenho quando a quantidade de soluções dispersas do conjunto $P$ (PSize) é igual a 100 , ou seja, para os diferentes valores de $b$ e $b_{1}$ utilizados nas simulações, a solução média ficou mais próxima da solução otimizada quando utilizado PSize $=100$ superando, em todos os casos, os testes feitos com PSize $=150$. As Tabelas IX e X apontam também que apesar do melhor resultado ter sido obtido com $b$ $=14$ e $b_{1}=7$ (Tabela IX) que encontrou a solução ótima 7067807 nas vinte iterações obtendo coeficiente de variação de $0 \%$, os parâmetros que obtiveram um melhor desempenho para os diferentes critérios de convergência foram $b=12$ e $b_{1}=6$, como pode ser observado na Tabela IX para o valor de PSize $=150$ e na Tabela X para os dois valores de PSize.

Na Tabela XI apresentam-se os custos totais da solução otimizada do problema de PSDEE obtida pela BD para a rede de 54 barras apresentada na tabela XII.

A solução otimizada apresentada pela BD não viola a restrição de tensão (tensão mínima de 0.981 pu) e de corrente nos condutores (corrente máxima de 380 A). A capacidade demandada de cada subestação ao final do horizonte de planejamento para o cenário com fator de carregamento de $100 \%$ é apresentada na Tabela XIII. 


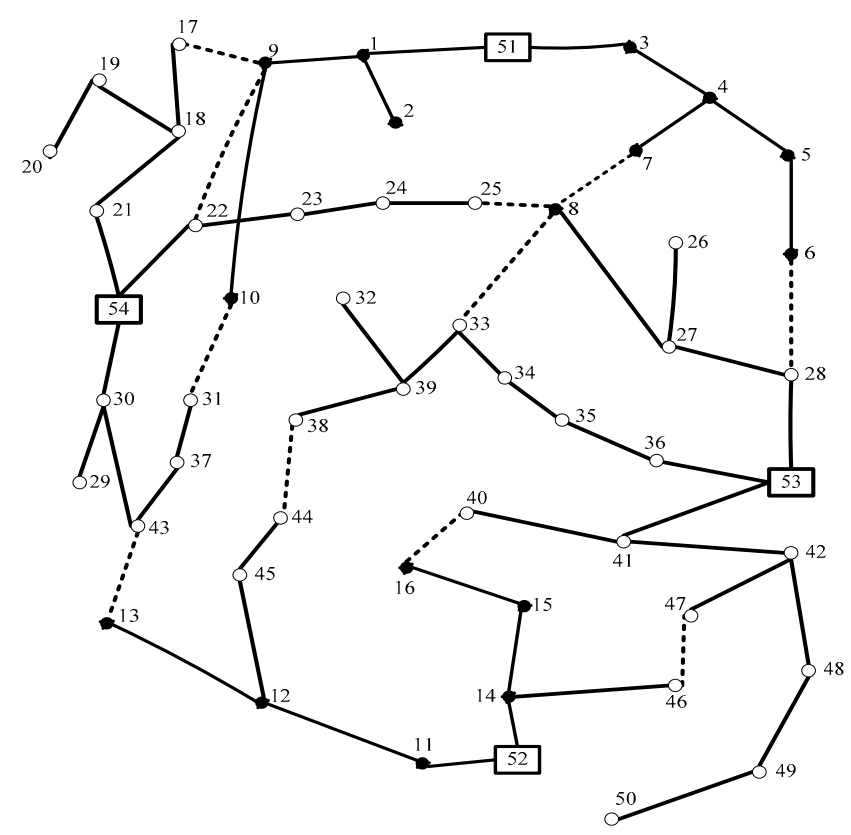

Figura 4. Topologia otimizada obtida pela BD para o sistema de 54 barras.

TABELA XI. CUSTOS TOTAIS DA SOLUÇÃO OTIMIZADA DA REDE DE 54 BARRAS FORNECIDOS PELA BD.

\begin{tabular}{lccc}
\hline & \multicolumn{4}{c}{ Custos (US\$) } \\
Linhas & SE & Perdas & Total \\
\hline 565210 & 6000000 & 502597 & 7067807 \\
\hline
\end{tabular}

TABELA XII. SOlUÇÃO OTIMIZADA OBTIDA PELA BD PARA O SISTEMA DE 54 BARRAS.

\begin{tabular}{cc|cc|cc|cc|cc}
\hline$i$ & $T C_{i}$ & $i$ & $T C_{i}$ & $i$ & $T C_{i}$ & $i$ & $T C_{i}$ & $i$ & $T C_{i}$ \\
\hline 1 & 4 & 12 & 2 & 23 & 1 & 36 & 1 & 50 & 4 \\
2 & 2 & 13 & 2 & 25 & 1 & 37 & 1 & 51 & 2 \\
3 & 2 & 14 & 2 & 26 & 1 & 40 & 1 & 52 & 4 \\
4 & 2 & 15 & 2 & 27 & 1 & 41 & 1 & 53 & 1 \\
5 & 2 & 16 & 2 & 29 & 1 & 43 & 1 & 55 & 2 \\
7 & 2 & 17 & 1 & 30 & 1 & 44 & 1 & 56 & 1 \\
8 & 2 & 18 & 1 & 31 & 2 & 45 & 1 & 57 & 1 \\
9 & 2 & 19 & 1 & 33 & 4 & 47 & 4 & 58 & 1 \\
10 & 2 & 21 & 2 & 34 & 1 & 48 & 4 & 59 & 1 \\
11 & 2 & 22 & 2 & 35 & 2 & 49 & 4 & 61 & 1 \\
\hline
\end{tabular}

TABELA XIII. POTÊNCIA EM CADA SUBESTAÇÃo DO SISTEMA DE 54 BARRAS AO FINAL DO HORIZONTE DE PLANEJAMENTO.

\begin{tabular}{ccc}
\hline Subestação & $\begin{array}{c}\text { Capacidade } \\
\text { (MVA) }\end{array}$ & $\begin{array}{c}\text { Capacidade da } \\
\text { demanda }(M V A)\end{array}$ \\
\hline 51 & 16.7 & 16.0 \\
52 & 16.7 & 11.5 \\
53 & 22.0 & 21.1 \\
54 & 22.0 & 17.6 \\
\hline
\end{tabular}

\section{B. Sistema de 417 barras}

Sistema de distribuição de $10 \mathrm{kV}$, com 417 barras, tendo duas subestações existentes que não permitem repotencialização e uma subestação candidata para ser construída com duas capacidades possíveis. A rede possui 88 circuitos em operação e 385 candidatos para expansão, além de 4 diferentes tipos de condutores que podem ser utilizados para instalação em novas linhas e/ou recondutoramento em linhas existentes. Os dados do sistema de 417 e sua configuração topológica inicial encontram-se em [14]. Os valores dos parâmetros utilizados para a BD nesse sistema foram os que apresentaram melhores resultados para a rede de 54 barras (PSize $=100, b=12$ e $b_{1}=5$ ) (Tabelas IX e X). Os valores dos parâmetros para o cálculo da dissimilaridade não sofreram alterações, apenas as constantes de penalidade das restrições 6,7 e 8 na função de adaptação foram modificados para 10000, 1000 e 100000, respectivamente. Na Tabela XIV apresenta-se a solução otimizada obtida pela BD. Esta solução não viola a restrição de tensão (tensão mínima de $0.95 \mathrm{pu}$ ) e de corrente nos condutores (corrente máxima de 333 A). As capacidades das subestações em MVA no final do horizonte de planejamento para o cenário com fator de carregamento de $100 \%$ estão apresentadas na Tabela XV.

TABELA XIV. CUSTOS TOTAIS DA SOLUÇÃo OTIMIZADA DA REDE DE 417 BARRAS FORNECIDOS PELA BD.

\begin{tabular}{cccc}
\hline \multicolumn{4}{c}{ Custos $\left(10^{3} \mathrm{US} \$\right)$} \\
Linhas & SE & Perdas & Total \\
\hline 1878,13 & 2000 & 624,19 & 4502,32 \\
\hline
\end{tabular}

TABELA XV. POTÊNCIA EM CADA SUBESTAÇÃO DO SISTEMA DE 417 BARRAS AO FINAL DO HORIZONTE DE PLANEJAMENTO.

\begin{tabular}{ccc}
\hline Subestação & $\begin{array}{c}\text { Capacidade } \\
\text { (MVA) }\end{array}$ & $\begin{array}{c}\text { Capacidade da } \\
\text { demanda }(M V A)\end{array}$ \\
\hline 417 & 8.0 & 7.9 \\
418 & 40.0 & 12.6 \\
415 & 40.0 & 10.4 \\
\hline
\end{tabular}

\section{CONClusões}

Neste trabalho apresentou-se uma metodologia para resolver o problema de planejamento de sistemas de distribuição de energia elétrica de média tensão através da implementação da metaheurística Busca Dispersa utilizando a codificação sequencial aditiva. Considera-se que a proposta desta metodologia é a principal contribuição deste trabalho uma vez que esta meta-heurística não foi ainda explorada na solução do problema de planejamento de sistemas de distribuição.

$\mathrm{Na}$ rede de 54 barras foram realizados diversos testes com diferentes valores dos parâmetros da BD como método de avaliação da influência dos mesmos na convergência do algoritmo. Além disso, apresentou-se a solução otimizada pela BD para a rede de 417 barras. Comparações com outros resultados apresentados na literatura especializada não puderam ser realizadas devido a ausência de informações, diferenças no modelo ou no tipo de planejamento considerado.

\section{REFERÊNCIAS}

[1] B. R. Pereira Junior, A. M. Cossi, J. Contreras and J. R. S. Mantovani, "Multiobjective multistage distribution system planning using tabu search", IET Generation, Transmission \& Distribution, v. 8, Iss. 1, p. $35-45,2014$

[2] A. Cossi, L. W. d. Silva, J. Mantovani and R. Romero, "Primary Power Distribution Systems Planning Taking Into Account Reliability, 
Operation and Expansion Costs", IET Generation, Transmission \& Distribution, v. 6, n. 3, p. 172-180, 2012

[3] I. J. Ramírez-Rosado and J. A. Dominguez-Navarro, "New Multiobjective Tabu Search Algorithm for Fuzzy Optimal Planning of Power Distribution Systems" IEEE Transactions on Power Systems, New York, v. 21, n. 1, p. 224-233, February, 2006.

[4] J. L. Bernal-Agustín and I. J. Ramírez-Rosado, "Genetic Algorithm Applied to the Design of Large Power Distribution Systems", IEEE Transactions on Power Systems, New York, v. 13, n. 2, p. 696-703, May, 1998.

[5] V. Miranda, J. V. Ranito and L. M. Proença, "Genetic Algorithms in Optimal Multistage Distribution Network Planning”, IEEE Transactions on Power Systems, New York, v. 9, n. 4, p. 1927-1933, November, 1994.

[6] E. G. Carrano, R. H. C. Takahashi, R. R. Saldanha, L. A. E. Soares and O. M. Neto, "Electric Distribution Network Multiobjective Design Using a Problem-specific Genetic Algorithm", IEEE Transactions on Power Systems, New York, v. 21, n. 2, p. 95-105, April, 2006.

[7] F. Mendoza, J. L. Bernal-Agustín and J. A. Dominguez-Navarro, "Nsga and Spea Applied to Multiobjective Design of Power Distribution Systems". IEEE Transactions on Power Systems, New York, v. 21, n. 4, p. 1938-1945, November, 2006.

[8] J. F. Gómez, H. M. Khodr, P. M. Oliveira, L. Ocque, J. M. Yusta, R. Villasana and A. J. Urdaneta, "Ant Colony System Algorithm for the Planning of Primary Distribution Circuits". IEEE Transactions on Power Systems, New York, v. 19, n. 2, p. 996-1104, May, 2004.

[9] E. G. Carrano, F. G. Guimaraes, R. H. C. Takahashi, O. M. Neto and F. Campelo, "Electric Distribution Network Expansion Under Loadevolution Uncertainty Using an Immune System Inspired Algorithm", IEEE Transactions on Power Systems, New York, v. 22, n. 2, p. 851861, May, 2007.

[10] C. Rego and P. Leão, "A Scatter Search Tutorial for Graph-based Permutation Problems". School of Business Administration, The University of Mississippi: Working Paper Series, Hearin Center for Enterprise Sciences, 2002.

[11] M. Laguna and R. Martí,Scatter Search: Methodology and Implementations in C. Kluwer Academic Publishers, Norwell, 2002.

[12] H. D. de M. Braz and B. A. de Souza, "Distribution Network Reconfiguration Using Genetic Algorithms With Sequential Encoding: Subtractive and Additive Approaches", IEEE Transactions on Power Systems, v. 26, n. 2, p. 582-593, May, 2011.

[13] T. Gönen, Electric Power Distribution System Engineering. New York, McGraw-Hill, 1986.

[14] J. L Bernal-Agustín, "Application Of Genetic Algorithms to the Optimal Design of Power Distribution Systems", Tese (Ph. D. in Electrical Engineering) University of Zaragoza, 1998.

[15] D. Shirmohammadi and H. W. Hong, "Reconfiguration of Electric Distribution Networks for Resistive Line Losses Reduction", IEEE Transactions on Power Delivery, v. 4, n. 2, p. 1492-1498, 1989.

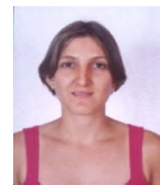

Súzan Grazielle Benetti de Pádua é graduada em Matemática pela Universidade do Estado de Mato Grosso (UNEMAT), Barra do Bugres, Mato Grosso, Brasil, em 2004. Obteve o título de mestre em Matemática pela Universidade Estadual de Campinas (UNICAMP), Campinas, São Paulo, Brasil, em 2008 e de Doutor, em Engenharia Elétrica pela Universidade Estadual Paulista (UNESP), Ilha Solteira, São Paulo, Brasil, em 2014. Atualmente é professor da Universidade do Estado de Mato Grosso (UNEMAT) e suas pesquisas se concentram na área de planejamento de sistemas de distribuição e otimização.

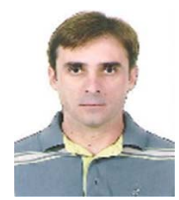

Antonio Marcos Cossi é, graduado em Engenharia Elétrica pela Universidade Estadual Paulista "Júlio de Mesquita Filho" (UNESP), Ilha Solteira, São Paulo, Brasil, em 2000. Obteve o título de Mestre e Doutor Universidade Estadual Paulista "Júlio de Mesquita Filho" (UNESP), Ilha Solteira, São Paulo, Brasil, em 2000 e 2008, respectivamente. Atualmente é professor e pesquisador no Departamento de Matemática da Universidade Estadual Paulista "Júlio de Mesquita Filho" (UNESP), Ilha Solteira, São Paulo, Brasil. Suas pesquisas se concentram nas áreas de Planejamento e Controle de Sistemas de Energia Elétrica, atuando principalmente nos seguintes temas: Planejamento, confiabilidade e qualidade do fornecimento de energia elétrica, de sistemas de distribuição de energia elétrica.

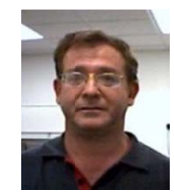

José Roberto Sanches Mantovani é, graduado em Engenharia Elétrica pela Universidade Estadual Paulista "Júlio de Mesquita Filho" (UNESP), Ilha Solteira, São Paulo, Brasil, em 1981. Obteve o título de Mestre e Doutor pela Faculdade de Engenharia Elétrica e de Computação da Universidade Estadual de Campinas (UNICAMP), Campinas, São Paulo, Brasil, em 1987 e 1995, respectivamente. Atualmente é professor titular do Departamento de Engenharia Elétrica da Universidade Estadual Paulista "Júlio de Mesquita Filho" (UNESP), Ilha Solteira, São Paulo, Brasil. É membro do IEEE (Member IEEE). Suas pesquisas se concentram nas áreas de Planejamento e Controle de Sistemas de Energia Elétrica, atuando principalmente nos seguintes temas: Desenvolvimento de modelos matemáticos e aplicação de técnicas metaheurísticas e de otimização clássica em problemas de planejamento controle de sistemas de geração, transmissão e distribuição de energia elétrica, confiabilidade e qualidade do fornecimento de energia de sistemas de distribuição de energia elétrica. 